\title{
Aplicación de las unidades fraseológicas a la enseñanza de E/LE
}

\author{
ANDREA GARCÍA MONTES \\ Universitat de València \\ angarmo4@alumni.uv.es \\ ANDREA LA Hoz MiRAlLES \\ Universitat de València \\ anlahoz@alumni.uv.es \\ LIDIA LATORRE GARCÍA \\ Universitat de València \\ lilagar@alumni.uv.es \\ Miguel LóPez MARTíneZ \\ Universitat de València \\ milomar2@alumni.uv.es
}

Resumen: El objetivo de este artículo es señalar que se puede acercar el aprendizaje de las unidades fraseológicas a la clase de E/LE en los niveles iniciales (A1-A2). La dificultad que puede suponer este tipo de estructuras hace que los diferentes manuales de E/LE no las recojan hasta llegado un nivel B2. Tras reparar en esta situación, hemos decidido elaborar una serie de actividades, con carácter lúdico, para poder utilizarlas en clase con los alumnos e intentar que aprendan diferentes locuciones mediante juegos. Para el diseño de estas actividades hemos delimitado nuestro corpus de unidades fraseológicas siguiendo una metodología basada en la noción de metáfora conceptual. Creemos que si los alumnos empiezan a utilizar unidades fraseológicas desde el principio del aprendizaje les será más fácil su reconocimiento y adquisición en niveles superiores.

Palabras clave: unidades fraseológicas, enseñanza, actividades E/LE, niveles iniciales.

\begin{abstract}
The aim of this paper is to show that phraseological units can be taught in the initial levels (A1-A2) of E/LE. These units are complex for these levels, because of that they don't appear in many handbooks until B2 level. Once we realized this situation, we have decided to prepare different ludic activities for using them in the classroom and trying to teach the students plenty of these units while they are having a good time. For designing these activities, we have delimited our corpus of phraseological units following a conceptual metaphor based methodology. From our point of view, we consider that the early use of the phraseological units will benefit the students because they will be able to recognize them and use them in superior levels.
\end{abstract}

Keywords: phraseological units, teaching, E/LE activities, initial levels. 


\section{Introducción}

El acercamiento de las unidades fraseológicas (UF) a los niveles iniciales de enseñanza de español como lengua extranjera (E/LE) es una idea apenas contemplada hasta el momento. Si bien es cierto que su aprendizaje en los niveles superiores de diversas lenguas ha ido adquiriendo peso en los últimos años, pocos son los autores que se han posicionado por su enseñanza en los niveles iniciales. Según la definición ofrecida por Corpas, las UF son:

[...] combinaciones estables formadas por al menos dos palabras y cuyo límite superior se sitúa en la oración compuesta. Se caracterizan por la alta frecuencia de aparición en la lengua y de coaparición de sus elementos integrantes, así como por la institucionalización, la estabilidad, la idiomaticidad y la variación potencial que dichas unidades presentan en diverso grado.

Corpas (1996: 23)

Así pues, las UF son expresiones idiomáticas, es decir, son construcciones lingüísticas cuya significación no es composicional. Forman parte del léxico de una lengua y su enseñanza suele seguir un método audio-lingual, esto es, mediante la repetición de una lista de elementos. $\mathrm{y}$

Dado su carácter idiomático, la complejidad para comprender el significado de las UF supone un problema para los estudiantes de lengua extranjera. En su primera toma de contacto con cualquiera de estas expresiones, es habitual que los aprendientes realicen una traducción literal carente de sentido, la cual no funciona en el contexto de uso. Por este motivo, ya que su frecuencia de aparición en el discurso del habla coloquial es alta, creemos necesario considerar cómo enseñarlas - mediante la introducción de actividades de carácter lúdico en el aula - en los niveles iniciales de E/LE para que su aprendizaje resulte efectivo.

\section{Delimitación del objeto de estudio}

Nuestro objeto de estudio son las expresiones idiomáticas o UF. Como detallaremos en $\S$ 3 , para este artículo hemos seleccionado UF relacionadas con el proceso de aprendizaje y la (auto)evaluación del alumnado.

\subsection{Justificación}

Tras el estudio y revisión de diversos manuales de enseñanza de español para extranjeros, así como de manuales de español especializado, no encontramos reflejadas las UF hasta llegado un nivel B2, en el que se presentan como listas de elementos léxicos y se propone su aprendizaje - como meras expresiones fijas - mediante procesos mnemotécnicos. Además, en el Marco Común Europeo de Referencia del Centro Virtual Cervantes no se pide la competencia de estas unidades hasta los niveles B2-C1 y no se plantea su enseñanza para niveles más básicos.

Con esta experiencia práctica pretendemos demostrar que, si se trata su uso en los niveles A1 y A2 con la metodología adecuada, su aplicación de forma ascendente será factible. Esta propuesta, que esperamos resulte útil en la enseñanza de lengua española, puede ser adaptable a cualquier idioma. 


\subsection{Objetivos generales y específicos}

Nuestra finalidad es acercar las UF a los niveles A1 y A2. Para alcanzarla nos centraremos en las unidades más transparentes, puesto que son más accesibles para los estudiantes de dichos niveles. Además, procuraremos introducirlos en un contexto de uso real, así como en el ámbito coloquial de la L2. De este modo, se amplía su repertorio léxico y, por lo tanto, se promueve el desarrollo tanto de sus destrezas orales como escritas.

Con las diferentes actividades aquí planteadas pretendemos evitar la posible fosilización de los errores y potenciar las inteligencias múltiples. Las actividades han sido elegidas para activar en mayor o menor medida cada una de las capacidades expuestas en la teoría de Howard Gardner (1983) en su libro Frames of Mind: The Theory of Multiple Intelligences, la cual indica que existen diferentes tipos de inteligencias (lingüísticaverbal, lógica-matemática, visual-espacial, musical, kinestésica-corporal, intrapersonal, interpersonal y naturalista), todas ellas autónomas e interrelacionadas a su vez.

\section{Metodología y corpus}

\subsection{Metodología}

Para el diseño de este corpus se ha hecho una reflexión desde la semántica cognitiva (Lakoff y Johnson, 1987; Cuenca y Hilferty, 1999) atendiendo a la capacidad de corporeización del cognitivismo, en la que se relaciona el lenguaje con nuestra experiencia corpórea, física, social y cultural. Consideramos que la vivencia de experiencias conectadas con el alumno potencia su aprendizaje. Debido a que las UF tienen un significado abstracto, nos hemos servido de metáforas conceptuales: estructuras concretas y físicas que ordenan dominios abstractos. Mediante el estudio de estas metáforas se observa que un gran número de expresiones lingüísticas tienen su significado en un dominio determinado, mientras que toman su significante de otro dominio distinto. Esta relación entre dominios es la que ayuda a conectar conceptos abstractos con concretos, de manera que en el aprendizaje de una L2 resultan útiles para acabar con el aprendizaje audio-lingual y conectar con las experiencias del alumno. Las correlaciones entre dominios que hemos establecido para nuestro corpus han sido las siguientes:

\begin{tabular}{|l|l|}
\hline \multicolumn{1}{|c|}{ DOMINIO ORIGEN } & \multicolumn{1}{c|}{ DOMINIO META } \\
\hline Situación agradable & Aprendizaje \\
\hline $\begin{array}{l}\text { Estar entre una situación agradable y } \\
\text { otra desagradable }\end{array}$ & Aprendizaje mejorable \\
\hline Situación desagradable & Aprendizaje fallido \\
\hline Superar una situación crítica & Esfuerzo \\
\hline Más posesión & Más control \\
\hline Menos posesión & Menos control \\
\hline
\end{tabular}

Tabla 1. Correlaciones de dominios 


\subsection{Diseño del corpus}

Teniendo en cuenta la finalidad didáctica y el contexto de aprendizaje en el que nos encontramos en el aula, hemos considerado apropiado limitar nuestras unidades a conceptos relacionados con el proceso de aprendizaje y con la (auto)evaluación. Es necesario que estas unidades sean simples, pues así aseguramos su aplicación para cualquier aprendiente de los niveles A1-A2.

Atendiendo a los dominios planteados en $\S 3.1$ se ha creado una tabla en la que se ordenan las UF elegidas para este trabajo, de manera que todas las unidades entran dentro de una metáfora conceptual, que conecta el significado abstracto con una experiencia física y concreta que resulte accesible para el alumno. De esta forma se potencia su aprendizaje y se conoce su estructura, teniendo en cuenta cómo se generan significados y se vinculan dentro de la semántica cognitiva. El corpus que hemos empleado para nuestras actividades es el siguiente:

\begin{tabular}{|c|c|c|c|c|c|}
\hline $\begin{array}{c}\text { BIEN } \\
\text { (SITUACIÓN } \\
\text { AGRADABLE) }\end{array}$ & $\begin{array}{c}\text { REGULAR } \\
\text { (ESTAR ENTRE } \\
\text { UNA SITUACIÓN } \\
\text { AGRADABLE Y } \\
\text { UNA } \\
\text { DESAGRADABLE) }\end{array}$ & $\begin{array}{c}\text { APRENDIZAJE } \\
\text { FALLIDO } \\
\text { (SITUACIÓN } \\
\text { DESAGRADABLE) }\end{array}$ & $\begin{array}{c}\text { ESFUERZO } \\
\text { (SUPERAR } \\
\text { UNA } \\
\text { SITUACIÓN } \\
\text { CRÍTICA) }\end{array}$ & $\begin{array}{l}\text { CONTROL } \\
\text { (POSESIÓN) }\end{array}$ & $\begin{array}{l}\text { NO CONTROL } \\
\text { (NO POSESIÓ) }\end{array}$ \\
\hline $\begin{array}{c}\text { Estar como } \\
\text { pez en el agua }\end{array}$ & Ni fu ni fa & Meter la pata & $\begin{array}{l}\text { Ir contra } \\
\text { viento y } \\
\text { marea }\end{array}$ & $\begin{array}{c}\text { Tener la } \\
\text { sartén por el } \\
\text { mango }\end{array}$ & Perder el hilo \\
\hline $\begin{array}{c}\text { Estar en tu } \\
\text { salsa }\end{array}$ & Regulín regulán & Dar calabazas & $\begin{array}{c}\text { Luchar con } \\
\text { uñas y } \\
\text { dientes }\end{array}$ & $\begin{array}{c}\text { Coger el } \\
\text { toro por los } \\
\text { cuernos }\end{array}$ & $\begin{array}{c}\text { Perder las } \\
\text { riendas }\end{array}$ \\
\hline $\begin{array}{l}\text { ir sobre } \\
\text { ruedas }\end{array}$ & & & & & \\
\hline
\end{tabular}

Tabla 2. Corpus de unidades manejado

\section{Actividades}

Para alcanzar los objetivos planteados, hemos diseñado una serie de actividades que pueden desarrollarse a lo largo de las diferentes unidades didácticas. En nuestro caso, las hemos diseñado utilizando un corpus acotado de unidades (véase $\S 3.2$ ), pero se puede aplicar cualquier otro conjunto de UF.

Para nosotros, una unidad didáctica consta del siguiente tipo de actividades $^{l}$ : de calentamiento, de andamiaje, actividades prácticas, la tarea final y actividades de (auto)evaluación. De acuerdo con estas, clasificaremos nuestras actividades según el tipo al que se correspondan.

\footnotetext{
1 Estos términos equivaldrían a los propuestos por Gelabert, Bueno y Benítez (2002): tareas de preactividad (calentamiento), tareas de postactividad (andamiaje, prácticas y tarea final) $\mathrm{y}$ autoevaluación.
} 


\subsection{Actividades de calentamiento}

Las tareas de calentamiento consisten en presentar de forma atractiva a nuestros alumnos el tema que se va a desarrollar en la unidad. En el caso de las UF, con el desarrollo de las actividades del «Mercado de expresiones» y del «Puzle de las expresiones», pretendemos hacer evidente a nuestros aprendientes de A1-A2 que este tipo de expresiones, que en principio parecen complicadas y extrañas, existen en sus respectivas lenguas maternas.

Con «Mercado de expresiones», pensada para tener una duración máxima de 30 minutos, se trabaja la comprensión oral y escrita, ya que consiste en hacerles pensar y reflexionar acerca de las UF que más utilizan en su lengua de origen. De esta forma, tras darles algunos minutos, tienen que exponer al resto de compañeros cuál es la expresión que han pensado y cómo creen que se dice en español. Así, ayudamos a activar tanto la inteligencia intrapersonal (al pensar su UF) como la interpersonal (en el momento de compartir con el resto la unidad) y la lingüística-verbal.

Por su parte, en «Puzle», que puede tener una duración de hasta 15 minutos, el profesor entrega varias piezas de puzle con UF escritas en ellas en diferentes idiomas. Los materiales necesarios para realizar esta actividad son cartulinas o cualquier otro material que pueda recortarse en forma de puzle. La actividad consiste en que por grupos de cuatro personas los alumnos tendrán que agrupar las UF que signifiquen lo mismo. En un primer momento, el profesor repartirá una pieza por equipo y un representante de cada grupo deberá levantarse a por las piezas que encajen, que estarán sobre la mesa del profesor, con la suya; si escoge una que no se ajusta deberá dejarla otra vez en el sitio y probar con otra distinta. El juego es en gran medida intuitivo, ya que a pesar de no conocer la expresión pueden llegar a completar el puzle gracias a la forma de las piezas. El objetivo principal es que los estudiantes se den cuenta de que este tipo de expresiones están presentes en todas las lenguas, que en ocasiones podemos encontrar equivalentes mientras que en otros casos resultan intraducibles. Podrán comprobar por su propia experiencia que, en algunos casos, las lenguas utilizan los mismos referentes, pero en otros son totalmente distintos. Por lo tanto, trabajaremos la comprensión escrita, la expresión oral y la interacción oral. En este sentido, las inteligencias que activaríamos serían las siguientes: inteligencia lingüística-verbal, visual-espacial (al ver escritas las expresiones en piezas de puzle), kinestésica-corporal (cuando se levanten a por las piezas) y la interpersonal (al trabajar en grupo).

\subsection{Actividades de andamiaje}

En el caso de las actividades de andamiaje, estas se basan en ofrecer modelos de aprendizaje a los estudiantes. De esta forma se empieza a consolidar el conocimiento que poseen sobre la unidad. Proponemos para ello el uso de Quizlet. Los materiales que se necesitan en esta actividad son una conexión a internet y cualquier dispositivo que pueda conectarse a la red.

La herramienta informática Quizlet nos sirve para repasar los conocimientos que se han adquirido en clase sobre las UF de una forma visual y auditiva. El uso de Quizlet está pensado para que se realice en casa y sea individual, por lo que no hay un tiempo establecido para realizar esta actividad de andamiaje. Para ello, el profesor proporciona el enlace de la unidad que haya preparado en Quizlet a sus alumnos. Esa unidad debe 
estar formada por al menos cinco expresiones sobre las que realizar las diferentes actividades que propone la plataforma (rellenar huecos, test, unir...). Gracias a estas actividades se desarrollan la inteligencia lingüística-verbal, la inteligencia lógicamatemática (actividades de unir) y la inteligencia visual-espacial (con las fichas que la plataforma ofrece). Además, estaríamos trabajando la comprensión escrita y oral, ya que se pueden escuchar las unidades que presente el profesor.

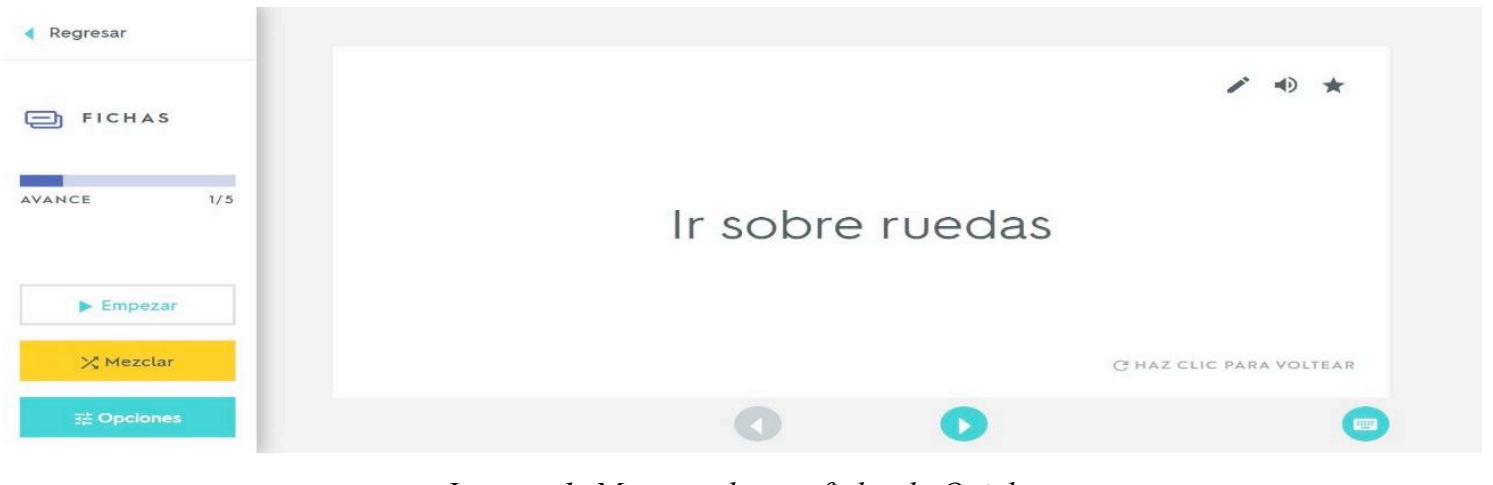

Imagen 1. Muestra de una ficha de Quizlet

Para llevar un seguimiento y saber si los estudiantes han utilizado esta herramienta en casa, el profesor puede, o bien tener una cuenta premium, y así poder ver los avances, o bien pedir que creen su propia unidad con las expresiones que más les gusten o les resulten más difícil de aprender.

\subsection{Actividades prácticas}

Las actividades prácticas están diseñadas para poner en funcionamiento los conocimientos que adquieren durante la unidad los estudiantes. En este caso proponemos la utilización de un Kahoot con UF y el «Rosco de Pasapalabra» como actividades prácticas.

En primer lugar, podemos emplear nuestro Kahoot con UF al principio de la clase o al final, con una duración de entre 10-15 minutos. En esta ocasión, al igual que en la actividad del Quizlet, se necesita una conexión a Internet y un dispositivo desde el que se pueda acceder a internet. Antes de empezar, el docente pregunta si quieren realizar el juego por parejas o por separado. Una vez organizada la clase, se da el código a los alumnos para acceder. Es interesante que, durante el juego, después de ver los errores, los alumnos expliquen con sus propias palabras lo que para ellos significan esas UF.

En segundo lugar, con el «Rosco de Pasapalabra» el profesor forma grupos de 3 o 4 personas y se les proyecta una imagen con las letras del abecedario. Por cada una de estas letras hay una pregunta relacionada con una unidad fraseológica trabajada previamente en clase. Una vez la pregunta haya aparecido, el grupo tiene un tiempo limitado para ponerse de acuerdo y responder. Gana el equipo que más respuestas acierte. En el caso de que un equipo falle la pregunta, existe la opción de rebote a otro equipo y de que este gane puntos extra si da la respuesta acertada. En esta actividad la duración puede extenderse hasta los 20 minutos y el único material necesario es un PowerPoint realizado por el docente, que proyectará en clase con las diferentes UF que vaya a trabajar. 
Estas dos actividades pretenden fomentar la expresión oral, la comprensión oral, la comprensión lectora y la interacción oral de nuestros aprendientes. Tras finalizar estas actividades podría realizarse unos minutos de debate para saber qué expresiones les han parecido difíciles, cuáles les han gustado $u$ otras cuestiones. De esta forma, las inteligencias que trabajaríamos con ambas son la inteligencia lingüística-verbal, la inteligencia visual-espacial (imágenes del Kahoot y del rosco) y la inteligencia interpersonal (por compartir sus opiniones y trabajar en grupos).

\subsection{Tarea final: «Del dicho al hecho...»}

La tarea final es el momento en el que los estudiantes plasman los conocimientos adquiridos a lo largo de la unidad didáctica o de un conjunto de clases. Es una herramienta para fijar los contenidos y a la que se dedica mayor tiempo, puesto que su éxito depende de la implicación de profesor y alumnos. En nuestro caso, la tarea gira en torno a los significados literales de las expresiones que hemos aprendido. Para ello, se divide al grupo en equipos de cuatro personas aproximadamente para grabar un vídeo corto - de no más de treinta segundos- representando el valor literal de una expresión. Cada uno de los miembros del grupo desarrolla una acción distinta, ya que no solo es necesario aparecer en el vídeo, sino también grabar, escribir el guion, escoger el material que se va a emplear, etc.

Con esta actividad pretendemos poner el foco de atención tanto en la inteligencia interpersonal como en la competencia oral de la interacción, dado que ambas son indispensables para llevar a cabo el vídeo. Además, su carácter lúdico favorece el aprendizaje de la expresión de su significado. La duración de esta tarea puede ser de una sola sesión o dividirse en más de una.

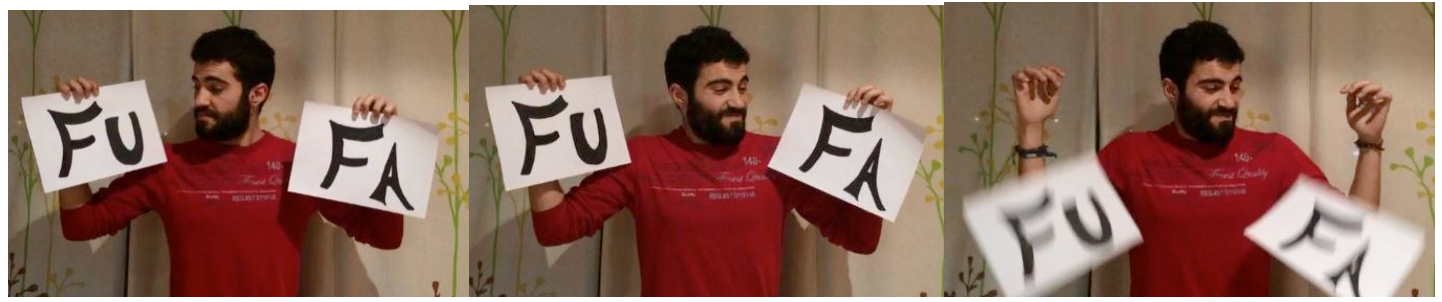

Imagen 2. Ejemplo de tarea final llevada a cabo con la expresión ni fu ni fa

\section{5. (Auto)evaluación}

Al comienzo de este trabajo teníamos como objetivo no solo enseñar las UF con actividades puntuales y concretas, sino también mediante su inmersión en el día a día del aula. Con esta finalidad, hemos diseñado una tabla de autoevaluación que permite a nuestros alumnos comprobar su propio progreso en diferentes ámbitos. Para ello, tendrán que marcar si su evolución es positiva (primera columna), si se puede mejorar (segunda columna) o si es negativa (tercera columna). 
Como observamos en la tabla siguiente ${ }^{2}$, podemos facilitar la comprensión de las expresiones mediante los colores y las imágenes. Estas, además, pueden cambiarse por otras una vez hayan sido adquiridas por los estudiantes. Lo más positivo de este ejercicio es la adquisición de la unidad a través del uso real y no a través de la memorización, como es aprendido tradicionalmente el vocabulario.

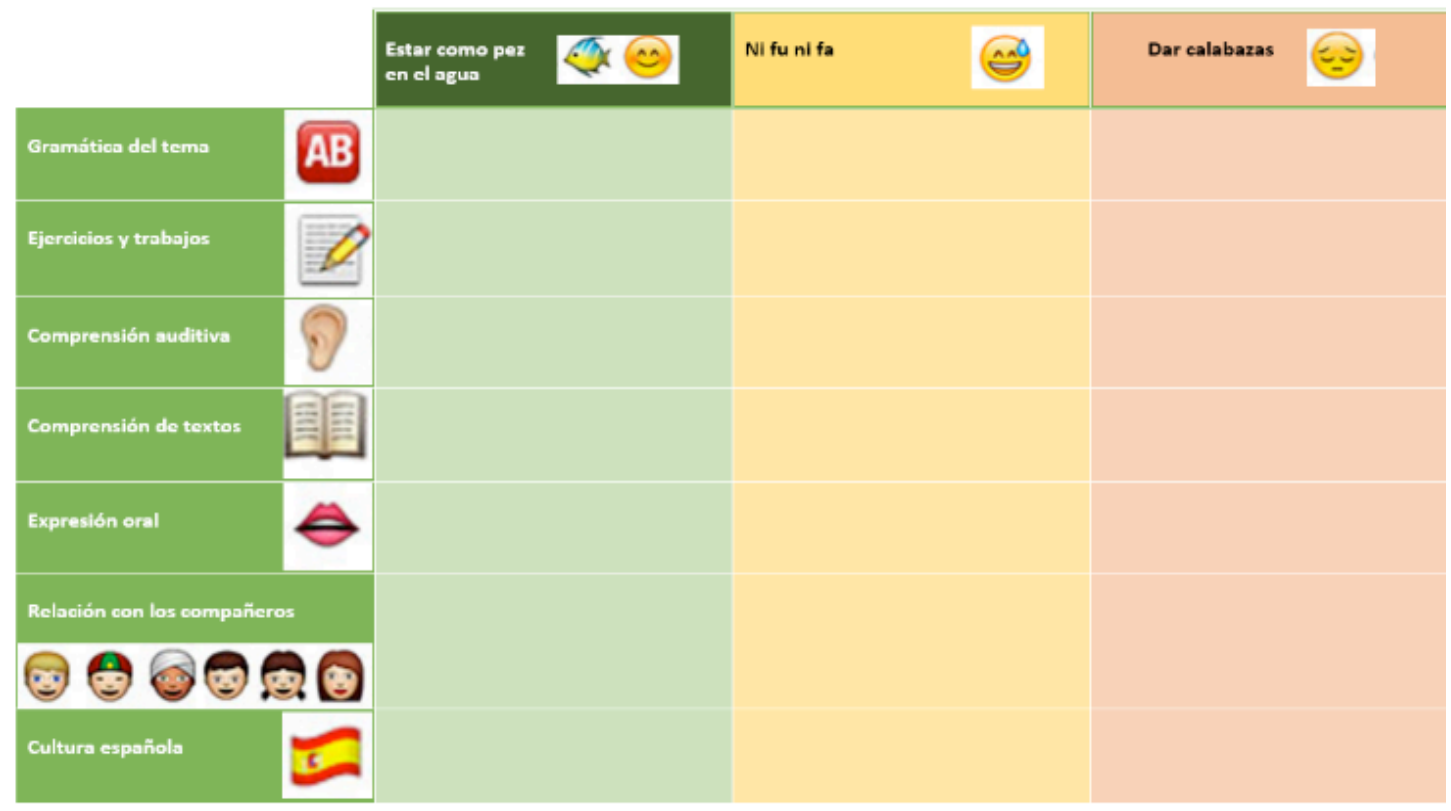

Imagen 3. Tabla de autoevaluación

2 Esta tabla de autoevaluación está pensada para aplicarse a una unidad didáctica en general, no necesariamente a una unidad en la que se trabajen únicamente las UF. Sin embargo, puede adaptarse a cualquier tipo de actividad si se modifican los ámbitos que el docente quiere evaluar. 


\section{Conclusiones}

Con lo visto en los anteriores apartados, podemos concluir que un acercamiento a las UF sí es posible, y además resulta útil, en los niveles iniciales, siempre y cuando nos sirvamos de actividades sencillas y lúdicas que incorporen las expresiones a su habla diaria.

Para ello, hemos desarrollado actividades de calentamiento, como «Mercado de expresiones»y «Puzle», de andamiaje mediante la herramienta informática Quizlet, prácticas mediante Kahoot y un «Rosco de Pasapalabra» y una tarea final «Del dicho al hecho...» para plasmar todo lo aprendido.

Una metodología centrada en la metáfora conceptual para la selección de las UF que pretendemos llevar al aula potencia su posterior aprendizaje, puesto que creamos vínculos entre conceptos accesibles al alumno y otros más alejados de su realidad.

Finalmente, todas las actividades aquí recomendadas pueden adaptarse a diferentes estudiantes y a diferentes niveles, empleando otras expresiones.

\section{Referencias bibliográficas}

CorPas Pastor, G. (1996). Manual de fraseología española. Madrid: Gredos.

CAlAÑas, J. A. (1999). «Los refranes en la enseñanza del alemán como lengua extranjera», en Paremia, 8, pp. 73-76. Recuperado el 12/04/17, de $<$ http://www.paremia2.site11.com/pdf/P8-11.pdf $>$.

Cuenca, M. J., y Hilferty, J. (1999). Introducción a la lingüística cognitive. Barcelona: Editorial Ariel.

GARDNER, H. (1983). Frames of Mind: The Theory of Multiple Intelligences. New York: Basic Books.

Gelabert, M. J., Bueno, I., y Benítez, P. (2002). Producción de materiales para la enseñanza de español. Madrid: Arco-Libros.

InSTITUTO CeRVANTES (2002). Marco común europeo de referencia para las lenguas: aprendizaje, enseñanza, evaluación. Madrid: Ministerio de Educación, Cultura y Deporte. Recuperado el 16/04/17, de <http://cvc.cervantes.es/obref/marco>.

LAKOFF, G., y Johnson, M. (2008). Metaphors we live by. Chicago: University of Chicago press. 